\title{
Usability of Venous Thromboembolism Prophylaxis Recommender System: A Pilot Study
}

\author{
Mehrdad KARAJIZADEH ${ }^{\mathrm{a}}$, Farid ZAND ${ }^{\mathrm{b}}$, Afsaneh VAZIN ${ }^{\mathrm{c}}$, \\ Roxana SHARIFIAN ${ }^{\mathrm{a}, 1}$ and Najmeh BAYATI ${ }^{\mathrm{b}}$ \\ a Health Human Resources Research Center, School of Management \& Medical \\ Information Sciences, Department of Health Information Management, Shiraz \\ University of Medical Sciences, Shiraz, Iran \\ ${ }^{\mathrm{b}}$ Anesthesiology and Critical Care Research Center, Shiraz University of Medical \\ Sciences, Shiraz, Iran \\ ${ }^{\mathrm{c}}$ Department of Clinical Pharmacy, Faculty of Pharmacy, Shiraz University of Medical \\ Sciences, Shiraz, Iran
}

\begin{abstract}
Poor usability of clinical decision support system impact negative on healthcare professionals, decrease usage and quality of clinical decision support system and result in a negative effect on patient outcome. Therefore, the objective of this study was the usability evaluation of the venous thromboembolism prophylaxis recommendation system. This study design is a pilot study. Totally seven individuals participate in the study that 4 out of 7 were ICU attending and 3 out of 7 were Residents in ICUs setting. System Usability Scale (SUS) was used to assess the usability of the clinical decision support system (venous thromboembolism prophylaxis recommendation system) integrated into the medication order entry system in the ICU setting. This study has shown that the mean System Usability Scale (SUS) score was 74.64. Summing up the results, it can be concluded that the usability quality of the venous thromboembolism prophylaxis recommendations system is good. Further research requires to evaluate the usability of the venous thromboembolism prophylaxis recommendation system by quantitative and qualitative methods in large scale.
\end{abstract}

Keywords. Usability, SUS, CDSS, VTE, Prophylaxis, Recommender System.

\section{Introduction}

Poor usability of clinical information systems was a global challenge in the healthcare information technology industry [1]. Poor usability of clinical information systems has a negative effect on healthcare professionals that due to burnout, low productivity, and alert fatigue [2]. The low productivity of health care professionals has negative effects on patient care. Furthermore, low-quality clinical information systems increase morbidity and mortality [3].

Previous studies have shown that the lack of involving the end-user in the clinical information system design process leads to poor usability [4]. Therefore, user-centered

${ }^{1}$ Corresponding Author, Roxana Sharifian, Health Human Resources Research Center, Almas Building, Alley 29, Qasrodasht Ave, Shiraz, Iran; Email: sharifianr@sums.ac.ir. 
design is essential to a well-designed clinical information system [5, 6]. Still, there is a large gap between the current clinical information system and physician tasks [7]. ISO defines usability as the" extent to which a system can be used by specified users to achieve specified goals with effectiveness, efficiency, and satisfaction in a specified context of use" [8]. To the authors' knowledge, no research has been investigated the usability of the venous thromboembolism prophylaxis recommendation system from the point of view of healthcare professionals in the ICU setting of Nemazee hospital. Therefore, in this paper, the usability level of the venous thromboembolism prophylaxis recommendation system was investigated. This study results could help to identify usability problems of the venous thromboembolism prophylaxis recommendation system and solve them.

\section{Methods}

This study design is a pilot study. The usability of the Venous Thromboembolism (VTE) prophylaxis recommendations system was evaluated in the ICUs setting of Nemazee Hospital in July 2021. Seven healthcare professionals were a participant in the study. 4 out of 7 were ICU attending and 3 of 7 were residents in ICUs. Venous thromboembolism prophylaxis recommendation system has been implemented in three ICUs (general ICU, central ICU, and emergency ICU) of Nemazee hospital. The usability of the VTE prophylaxis recommendation system has been assessed by System Usability Scale (SUS) questionnaire [9]. The data collection form had two parts: the first part included the SUS questionnaire items and the second part included a non-structured question about the usability of this system. The data collection form was attached as supplementary file 1 . System Usability Scale (SUS) questionnaire consists of 10 items with one of five responses that range from Strongly Agree to Strongly disagree. The usability score was calculated based on the Brooke study [9]. SUS Score above 68 is considered excellent usability based on the general guideline on interpretation SUS score. The sampling method was convenient because all physicians in the three ICUs settings were included. Each questionnaire item was described by mean and standard deviation. All statistical analyses were performed by SPSS 24 .

The study was approved by the local Institutional Review Board and ethics committee of Shiraz University of Medical Science (Approval ID: IR.SUMS.REC.1398.1046).

\section{Results}

These results have shown that use VTE prophylaxis recommendation system frequently, various functions in the VTE prophylaxis recommendations system were well integrated, and imagine that most people would learn to use the VTE prophylaxis recommendations system very quickly have a high mean (mean over 4.14) from respondent perspective. The mean System Usability Scale (SUS) Score was 74.64. Overall measurement results are summarized in Table 1. Only one participant had commented about the usability of this system ("It was difficult to move the form page up and down when using the system"). Other participants had no comment. 
Table 1. Descriptive Statistics Each Item of System Usability Scale (SUS) Questionnaire for VTE prophylaxis recommendation system

\begin{tabular}{lcc}
\hline \multicolumn{1}{c}{ System Usability Scale (SUS) } & Mean & S.D \\
\hline I think I would like to use the VTE prophylaxis recommendations system frequently. & 4.29 & 0.76 \\
\hline I found the VTE prophylaxis recommendations system unnecessarily complex. & 2.00 & 0.58 \\
\hline I thought the VTE prophylaxis recommendations system was easy to use. & 4.29 & 0.49 \\
\hline $\begin{array}{l}\text { I think that I would need the support of a technical person to be able to use the VTE } \\
\text { prophylaxis recommendations system. }\end{array}$ & 1.57 & 0.79 \\
\hline $\begin{array}{l}\text { I found the various functions in the VTE prophylaxis recommendations system were well } \\
\text { integrated. }\end{array}$ & 4.14 & 0.69 \\
\hline $\begin{array}{l}\text { I thought there was too much inconsistency in the VTE prophylaxis recommendations } \\
\text { system. }\end{array}$ & 1.71 & 1.11 \\
\hline $\begin{array}{l}\text { I would imagine that most people would learn to use the VTE prophylaxis } \\
\text { recommendations system very quickly. }\end{array}$ & 4.14 & 0.90 \\
\hline I found the VTE prophylaxis recommendations system very cumbersome to use. & 2.29 & 0.95 \\
\hline I felt very confident using the VTE prophylaxis recommendations system. & 3.43 & 1.72 \\
\hline $\begin{array}{l}\text { I needed to learn a lot of things before I could get going with the VTE prophylaxis } \\
\text { recommendations system. }\end{array}$ & 2.00 & 1.15 \\
\hline $\begin{array}{l}\text { System Usability Scale (SUS) Score } \\
\end{array}$ & 74.64 & 12.86
\end{tabular}

\section{Discussion}

This study indicated that the usability quality of the VTE Prophylaxis Recommendations System was good. To our best knowledge, this is the first study to investigate the usability of a homegrown venous thromboembolism prophylaxis recommendation system in the ICU setting of Nemazee hospital. These results concur with the other studies which have shown that clinical decision support system usability quality was good [10-12]. In contrast to some reports in the literature, there was usability quality of clinical information systems was low [7, 13]. This study indirectly implies that end-user involvement in the design of clinical decision support systems and homegrown clinical information systems improves the success and adoption rate of these systems. Also, multi-disciplinary collaboration increases the usability of clinical information systems.

The strength of our survey is that intensive care specialists assess the usability of the VTE Prophylaxis Recommendation System. The major limitation of this study is that the analysis does not enable to determine the usability of the VTE prophylaxis recommendation system by quantitate and qualitative methods in large scale, because this system implemented in ICUs of single center. Thus, the limitation of this study was the sample size. A further research requires to evaluate the usability of the VTE prophylaxis recommendations system by mixed methods (quantitate and qualitative methods) in large scale.

Summing up the results, it can be concluded that the usability quality of the VTE Prophylaxis recommendation system is good. Further research is required to evaluate the usability of the VTE Prophylaxis recommendation system by quantity and qualitative methods in on a large. In addition, iterative usability redesigns the process needs to improve the usability of the VTE Prophylaxis Recommendation System. 


\section{Acknowledgment}

This article was extracted from a doctoral thesis (research ID: 97-01-07-19299) supported by the Research Vice-Chancellor of Shiraz University of Medical Sciences. We are profoundly thanks the healthcare professionals at ICUs of Nemazee Hospital in Shiraz, Iran because this paper would not have been possible without their friendly collaboration.

\section{References}

[1] Staggers N, Xiao Y, Chapman L. Debunking health IT usability myths. Appl Clin Inform. 2013; 4(2):24150. doi: 10.4338/ACI-2013-03-IE-0016. PubMed PMID: 23874361.

[2] Guo J, Iribarren S, Kapsandoy S, Perri S, Staggers N. Usability Evaluation of An Electronic Medication Administration Record (eMAR) Application. Appl Clin Inform. 2011;2(2):202-24. doi: 10.4338/ACI2011-01-RA-0004. PubMed PMID: 23616871.

[3] Schulte F, Fry E. Death by 1,000 clicks: Where electronic health records went wrong. Kaiser Health News. 2019; 18.

[4] Rizvi RF, Marquard JL, Hultman GM, Adam TJ, Harder KA, Melton GB. Usability Evaluation of Electronic Health Record System around Clinical Notes Usage-An Ethnographic Study. Appl Clin Inform. 2017;8(4):1095-105. Epub 12/14. doi: 10.4338/ACI-2017-04-RA-0067. PubMed PMID: 29241247.

[5] Chou D. Health IT and patient safety: building safer systems for better care. Jama. 2012;308(21):2282-.

[6] Carayon P, Salwei ME. Moving toward a sociotechnical systems approach to continuous health information technology design: the path forward for improving electronic health record usability and reducing clinician burnout. Journal of the American Medical Informatics Association. 2021; 28(5): 10268 .

[7] Viitanen J, Hyppönen H, Lääveri T, Vänskä J, Reponen J, Winblad I. National questionnaire study on clinical ICT systems proofs: physicians suffer from poor usability. International journal of medical informatics. 2011;80(10):708-25.

[8] Available from: https://www.iso.org/obp/ui\#iso:std:iso:21902:ed-1:v1:en:term:3.19.

[9] Brooke J. SUS-A quick and dirty usability scale. Usability evaluation in industry. 1996;189(194):4-7.

[10] Genes N, Kim MS, Thum FL, Rivera L, Beato R, Song C, et al. Usability Evaluation of a Clinical Decision Support System for Geriatric ED Pain Treatment. Appl Clin Inform. 2016; 7(1):128-42. doi: 10.4338/ACI-2015-08-RA-0108. PubMed PMID: 27081412.

[11] Hudson D, Kushniruk A, Borycki E, Zuege DJ. Physician satisfaction with a critical care clinical information system using a multimethod evaluation of usability. International Journal of Medical Informatics. 2018;112:131-6. doi: https://doi.org/10.1016/j.ijmedinf.2018.01.010.

[12] von Dincklage F, Suchodolski K, Lichtner G, Friesdorf W, Podtschaske B, Ragaller M. Investigation of the usability of computerized critical care information systems in Germany. Journal of intensive care medicine. 2019;34(3):227-37.

[13] Bloom BM, Pott J, Thomas S, Gaunt DR, Hughes TC. Usability of electronic health record systems in UK EDs. Emergency Medicine Journal. 2021;38(6):410-5. doi: 10.1136/emermed-2020-210401. 